\title{
NOVENTA Y CINCO CASOS DE EMBARAZO ECTOPICO
}

\author{
Por el Doctor J. A. Gómez-Palacino (*)
}

1954

Esta es una síntesis de los casos de embarazo ectópico que se presentaron en el Instituto de Proteción Materna é Infantil de Bogotá desde el año de 1949 hasta el 31 de diciembre de 1953.

Nos abstendremos, porque ello le quitaria tal carácter de resumen, de hacer recapitulación de etiopatogenia, modalidades, variedades, etc. del embarazo ectópico; además, no hariamos otra cosa que repetir las descripciones de los textos y el hecho de presentar este trabajo ante una Sociedad de especialistas en Obstetetricia y Ginecologia, nos exime lógicamente de ello.

En los últimos cinco años han ingresado al Instituto 40.533 pacientes, habiéndose comprobado quirúrgicamente 95 casos de embarazo ectopico en evolución o complicados.

La inmensa mayoría, 93, han sido casos de gestación de pocas semanas: 6 a 12; el resto, es decir dos casos ha sido de embarazos ectópicos avanzados que hemos tenido la oportunidad de observar directamente y cuyas historias clinicas hemos incluido en forma separada por considerarlo de sumo interés.

Después de discriminar los citados 95 casos harcmos algunas observaciones y comentarios sobre algunos puntos que crecomos de importancia.

En el año de 1949 se hospitalizaron 7.367 pacientes, 23 de las cuales prescntaban embarazos ectópicos. Ello da un porcentaje de $0.31 \%$.

En 1950 ingresaron 7.352 pacientes, de las cuales 17 con embarazo ectópico. Porcentaje: $0.23 \%$.

En 1951, 8.109 pacientes y 20 casos de ectópico. Porcentaje: $0.23 \%$.

En 1952, 8.616 pacientes y 22 casos de cctopico. Porcentaje: $0.25 \%$.

En 1953, 9.223 pacientes y 13 casos de ectópico. Porcentaje: $0.14 \%$.

De los datos anteriores podemos concluír que la frecuencia de esta entidad se ha conservado semejante en el curso de los últimos cinco anos a excepción del año de 1953 en que la disminución fue notoria. Desde luego, no podramos hacer ninguna hipotesis al respecto, pero si llama la atención el hecho de que numerosos autores consideran que el embarazo ectópico ha aumentado su fre- 
cuencia a partir del empleo de los agentes quimioterápicos en el tratamiento de las afecciones ginecologicas y principalmente, anexiales. De aquí que este dato nos parezca contradictorio.

Por lo demás, el Dr. Enrique Archila ( ${ }^{1}$ ) en su tesis doctoral, sobre 24.597 historias consultadas dedujo un porcentaje de $061 \%$ al paso que el nuestro es tan sólo del $0.23 \%$.

El Dr. Humberto Góngora Sánchez (") encontró sobre un total de 2.846 gestantes 42 casos de embarazo ectopico para un porcentaje de $1.41 \%$ en el Hospital de Manizales.

Vale la pena tambien citar los casos intervenidos en el Servicio de Clínica Ginecológica del Ilospital de San Juan de Dios en donde el movimiento de pacientes es bastante considerable:

$$
\begin{aligned}
& \text { En 1949: } 14 \text { casos. } \\
& \text { En 1950: } 19 \text { casos. } \\
& \text { En 1951: } 8 \text { casos. } \\
& \text { En 1952: } 9 \text { casos. } \\
& \text { En 1953: } 10 \text { casos. }
\end{aligned}
$$

Comparando estos datos con los citados por autores extranjeros tenemos que: Bandl da un porcentaje de $0.005 \%$, W'inne da $1.3 \%$, Schuman encontró una incidencia de $0.33 \%\left({ }^{3}\right)$.

En cuanto a la edad de las pacientes afectadas hemos encontrado que para 9] casos el promedio de la edad ha sido de 29-15 años estando este dato de acuerdo con la gran mayoría de los autores que anotan la mayor frecuencia entre $\operatorname{los} 24$ y los 34 años. No se halló el dato de edad en cuatro de las historias clínicas consultadas.

Respecto a paridad anotamos:

20 casos se presentaron en pacientes primigestantes.

69 casos en pacientes con des o más embarazos.

6 casos $\sin$ dato de paridad registrado.

Hemos hallado 65 cason con antecedentes ginecológicos u obstétricos importantes: Menarquia tardia, trastornos mentruales (oligomenorrea, polimenorrea, monometrorragias, etc.), flujos inflamatorios, embarazos ectópicos anteriores, intervenciones previas sobre organos sexuales o intrapélvicos, abortos y partos prematuros.

En cuanto a localización hallamos lo siguiente:

55 casos se han implantado en el lado derecho.

28 casos en el izquierdo.

10 de los casos intervenides aparecen sin descripción quirúrgica al respecto.

Dos casos hallamos de embarazo ectópico bilateral.

No se ha registrado un sole caso en los últimos cinco años de embarazo ectópico coexistiendo con una gestacion tópica.

El promedio de tiempo de amenorrea ha sido de 58 días para 75 casos. Este dato faltó en 20 casos. 
En cuanto a sintomatologia hemos encontrado que en 82 de los casos figuró entre las manifestaciones el dolor; en 70 casos hubo hemorragia gevital; en 8 casos lipotimia; en 6, tenesmo rectal; en 6, vomito; 5 pacientes entraron en schock; 4 con retención de orina y 4 en anuria: Si tenemos en cuenta que el promedio de la tensión arterial fue d. 9. milimetres para la maxima y 58 milimetros para la mínima en los 95 casos y yue solo 5 pacientes entraron en estado de shock, he mos de concluir gue una de las causa de crore en el diagnóstico de embarazo ectopico complicado con ruptura, es el experar encontrar sicmpre el cuadro clínico correspondiente a la grosedad de la entidad cuando muy por el contrario, no es raro hallar pacientes con buena tension arterial, buen pulso e inclusive buen estado general en quicnes al practicar la laparotomia se encuentra una gran inundación intraperiotoneal y que de no haberse intervenido con oportunidad hubieran probablemente sucunbicto. Merece citarse en relación con lo anterior lo siguiente: 72 de nuestras pecientes presentaban-ruptura de su gestación ectópica.

De las 95 pacientes, $8($ ) se han intervenido con el diagnóstico de embarazo ectópico o haciendo parte dé las hipótesis más probables. Los casos restantes, han sido hallazgos operatorios. Creemos que es un índice de amplio margen de seguridad diagnóstica toda ve que en las pacientes hospitalarias la anamnésis es en la gran mayoría de los casos un verdadero problema; por lo demás, ingresan muchas veces tardiamente cuando el cuadro clinico se ha hecho en extremo confuso. Por otra parte, la ayuda de los análisis de laboratorio no fue siempre eficiente; muchas veces las pruebas biologicas fucron impracticables.

Crecmos que el índice diagnestico se puede mejorar en el futuro ayudán donos de algunos recursos que proco se emplean en al Instituto tales como la col potomía posterior de técnica fácil y de gran utilidad pero solo empleada en 7 de las pacientes de nuestra casuistica.

Las siguientes historias clinicas confirman nuestras aseveraciones:

Historia No 2.008 de 1951 :

Ingresa la paciente después de un aborto porque ha continuado sangrando. I. hemorragia se acompana de dolor permanente on el hipogastrio, disuria y tenesmo rectal, hay además temperatura de 37.8 grados $C$.

Examinada se hizo diagnóstico de pelviperitonitis postaborto. Con tal diag. nestico, se administró Penthotal para practicar luégo colpotomía con el fin de drenar el absceso. El resultado fue la obtención de abundante cantidad de sanger oscura.

En estas condiciones se practicó laparotomía encontrándose un embarazo tu. hinice derecho roto, siendo necesario practicar salpingoodorectomía pues fue imposible conservar el ovario. Naturalmente, la confusion diagnóstica retardó - Itatamiento adecuado. La paciente fue tratadas varios das con antibióticos, hirlo en el abdomen, etc. 


\section{Historia No 1.085 de 1953:}

Ingresa la paciente el 27 de mayo porque desde hace 8 días presenta dolores hipogástricos, expulsión de coágulos, vómito, constipación e hipertermia: 28.2 grados $\mathrm{C}$.

Al examen se encuentra: lengua saburral, timpanismo abdominal, dolor hipregástrico y sobre las fosas iliacas, defensa muscular no muy marcada. Al tacto 1. ginal se aprecia: cuello en situación posterior, largo, cerrado, fondos de saco wupados. Se hace el diagnóstico de pelviperitonitis y se instifuye el tratamiento inclicado en estos casos.

Veinticinco días más tarde la paciente se siente bien y pide la salida del Servicio pero cuatro días más tarde reingresa porque los dolores se han exacerbidto. En estas condiciones se hace nuevo examen y se practica colpotomía posterior; el resultado es la salida de sangre oscura. Se practica faparotomía y se encuentra un embarazo tubárico derecho roto; se hace salpingectomía. Hay una nota del Jefe de Clínica del Servicio que dice: "Es de anotar la discordancia de los síntomas clínicos y de diagnóstico con la gran inundación peritoneal".

Nos permitimos extraer de la tesis de grado del doctor Luis Novoa Rodrí. gue\% (op. cit.), la siguiente historia clinica del Scrvicio de Ginecología del Hos pital de San Juan de Dios:

Historia No 10.367 de 1952:

La paciente ingresa al Servicio el 9 de diciembre de 1952. Ultima regla a a lines de noviembre. La enfermedad se inició hace 8 dias con dolor en la fosa ilica izquicerda, vómito, escalofrio y malestar general.

El examen ginecológico revela: genitales externos normales. El espéculo permite apreciar un cuello de aspecto normal a travé del cual fluye hemorragia endecervical discreta. Al tacto vaginal el cuello se encuentra ligeramente blando, un poco desgarrado transversalmente y desviado a la izquierda; el cuerpo se en cuentra colocado en dextroposición. Los fondos de saco posterior y laterales ocu pates por una masa que abomba la pared posterior de la vagina: renitente, irregular mamelonada, muy dolorosa al tacto y que hace continuación a la tumefaccion abdominal. La histerotometría da 8 centimetros.

Impresion: a)-Pelviperitonitis TBC. b)--Proceso inflamatorio perviperitoncal. c)-Tumor degenerado del ovario izquierdo.

Posteriormente se practicó una punción del Douglas con fines diagnósticos y el resultado fue positivo para liquido sanguinolento.

Se intervino la enferma y se halló una masa de 10 centímetros de diámetro, encapsulada, que incluia trompa y ovario izquierdos adheridos al sigmoide y al Douglas. Se practicó salpingo-ooforectomía izquierda y se liberaron adheren cias en el anexo derecho. 
El diagnóstico de anatomía patológica fue: Embarazo uubario roto.

A manera de comentario de las dos últimas historias diremos que las intervenciones quirúrgicas se practicaron IIN MIES después de ingresar las enfermas al Hospital y luégo de practicar punciones del fondo de saco posterior. Debemos pensar en la importancia médica y secial de una intervención qui rúrgica practicada a tiempo; de ahi que nosotros deduzcamos que si las colpo tomias hubicran side hedas precozmente mucho se hubiera logrado en tiempo oportuno, con el consiguiente bendicio de las pacientes.

Respecto a la práctica de la colpotomia De lec (4) se manifiesta en la si guiente forma: "La punción exploradora del fondo de saco de Douglas es inoten siva, y a menudo presta gran utilidad. En los casos en que se sospecha un embarazo ectópico y en los muchos en que es dificil hacer con certeza el diagnóstico de gestación ectópica cón ruptura, se debería practicar la colpotomía posterior con mucho mayer frecuencia de lo que se hace en la actualidad".

En cuanto a las pruebas biológicas debemos anotar que ellas prestan una gran ayuda al diagnóstico y que el Instituto de Protección Materna e Infanti! cuenta actualmente con un servicio idóneo y oportuno, utilizándose de manera sistemática la reacción de Galli y Mainini de gran margen de seguridad y que permitirá en lo sucesivo mayor precisión diagnóstica.

Otros métodos de diagnóstico tales como el estudio anatomopatológico de los restos eliminados con la sangre uterina en los casos de duda respecto a si se trata de un embarazo ectópico o un aborto; en neumoperitóneo transabdominal y la peritoneoscopia, no han sido empleados entre nosotros.

En relación con los diagnósticos crrados y bajo los cuales se intervinieron 13 pacientes hemos encontrado:

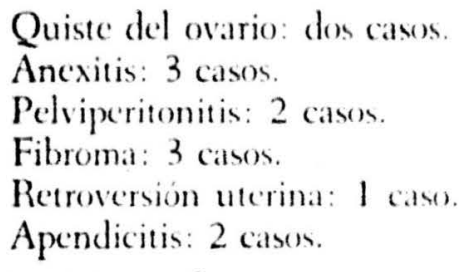

Sin diagnóstico se intervinieron dos paciontes.

Tratamiento.-Todos los casos resgistrados en este resumen fueron intervenidos quirúrgicamente por vía alta. Entre las intervenciones hemos en contrado:

60 salpingectomías.

12 sapingo-oforectomías.

I reseccion angulo intorsticial.

1 histerectomia fundica con resección del anexo izquierdo.

2 Resceciones parciales de ovario y sutura.

1 Resección parcial de la trompa.

Han faltado las descripciones de las operaciones practicadas en 18 casos. 
Coexistiendo con el embarazo ectópico se encontraron e intervinieron las siguientes entidades:

\author{
Quistes del ovario: 9 casos. \\ Quiste de la hidátide: 1 caso. \\ Retrodesviación uterina: I caso. \\ Fibromatosis uterina: I caso. \\ Hidrosalpinx: 1 caso. \\ Ancxitis: 1 caso.
}

Fueron practicadas también en el mismo acto quirúrgico, 7 apendicectomías.

A este respeceto vale la pena transcribir los siguientes conceptos de $\mathrm{Nu}$. biola $\left({ }^{8}\right)$ : "El apéndice, en toda operación por ectópico, debe ser respetado. No se olvide que la sangre constituye un excelente medio de cultivo para cualquier especie microbiana. Al hacer la apendicectomía, por mayores cuidados que se usen en su extirpación (aislamiento cuidadoso, operación fuera del abdomen y peritonización), siempre se movilizan focos microbianos, dotados de gran poder séptico".

De Lee (Op. cit.), afirma: $\mathrm{Si}$ al operar un embarazo ectópico "hubiere piosalpinx crónico en la otra trompa, estará bien extirparlo, pero no se intentará la escisión de ningún órgano. Es menos arriesgado no buscar NI EXTIRPAR en este momento el apéndice".

En las historias clínicas consultadas hemos encontrado 10 casos en los cuales luégo de practicar la intervención para ectópico roto, se han dejado drenes. Nos atrevemos a afirmar que tal conducta debe desecharse por cuanto con ello se expone a la paciente a una infección que sería siempre grave dadas las condiciones de inferioridad en que están aquellos organismos.

En varias intervenciones presenciadas por nosotros hemos visto que algu nos cirujanos acostumbran, en los casos complicados con ruptura, extracr los coágulos sanguíncos organizados para dejar a manera de autotransfusión la san gre líquida. Otros, desconocemos sus razones, desechan el procedimiento.

No tenemos noticia de que el sistema preconizado por Thies de extraer la sangre derramada para luégo filtrarla e inyectarla de nuevo, haya sido empleado entre nosotros. Por lo demás, según Argonz y Ricci ${ }^{6}$ ), se han visto casos de sepsis grave por este procedimiento y por ello, en otros medios tiende a desaparecer. De otra parte, en todo centro hospitalario debe funcionar un banco de sangre eficiente. Desde este punto de vista el Instituto Materno e Infantil cuenta con un servicio que satisface las necesidades; desafortunadamente las histo rias clínicas rara vez registran las transfusiones efectuadas y por ello no podemos dar un dato exacto.

Si bien es cierto que apenas cinco pacientes entraron en shock como consecuencia del estallido ovular y la hemorragia intraperitoneal, estamos de acuerdo con los diversos autores en que el concepto de que una paciente en estado de shock no se debe intervenir, no hace relacion con los estados de schock oca. sionados por la entidad de que nos ocupamos. Son pacientes que soportan re- 
lativamente bien el acto quirúrgico; además, la esperat a que la paciente se re cupere implica un riesgo quizá mayor que de da operación oportunamente practicada. Personalmente hemos presenciado actos quirúrgicos de esta índole y los resultados han sido francamente favorables. Sc ha seguido la conducta de aplicar soluciones dextrosadas hasta el momento en que se logra la hemóstasis; practicada ésta, se han hecho transfusiones de sangre suficientes. La aplicación de sangre antes, y sobre todo cuando cllo se hace a cierta velocidad tiene el incomeniente de aumentar la volemia y por consiguiente la presión en el foco hemorrágico dando por resultado el aumento de la perdida sanguinea y el des prendimiento de coagulos hemostáticos.

Anestesia.-Para las 86 de las intervenciones practicadas en el Instituto se empleo la anestesia general (ciclo-éter oxígeno) y solamente en 9 caso la anestesia espinal, algunos de ellos complitados con ruptura antigua:.

Sabiendo que el accidente agudo de la ruptura implica hipotensión, taquicardia y colapso periférico, es lógico pen ar que la raquianestesia esté contraindicada; además, en este tipo de interverfciones, la posición adecuada es la de Trendelemburg lo cual, agrega un riesgo más a la práctica de la anestesia espinal que podría interesar zonas medulares feligrosas. He ahí la razón por la cual, salvo circunstancias especiales (ruptura antigua del embarazo ectópico en pa(ientes en buenas condiciones generales, por ejemplo). la anestesia ideal es la zeneral por inhalación.

Pronóstico.-De la experiencia obtenida en los últimos cinco años en el Instituto Materno e Infantil, podemos afirmar que el embarazo ectópico com plicado o no, intervenido oportunamente y bajo los preceptos anotados en las pági nas anteriores, es de buen pronéstico respecto a la vida de la madre. Hacemos esta alirmación teniendo en cuenta el número de casos intervenidos en dicho medos hospitalario, en un plazo relativamente largo. De las 95 pacientes operadas po. demos decir que ninguna murio como consecuencia de tal entidad. Intortuna nedamente, hemos de registrar el caso de una paciente cuyos azares quirúgicos determinaron la muerte.

Se trata de la historia 670 de 1951. Esta paciente fue laparotomizada y al verilicar la exploración se comprobó la presencia de un embarazo tubárico iz quicrdo roto, salpingitis y ovario poliquístico derecho con matriz fibromatosa. En estas condiciones se realizo histerectomía subtotal; tresce días más tarde, fue neceesario practicar nueva laparotomía para extracr cuerpo extraño; tres dias después, se hizo aparente una fistula estercoral.

Las condiciones de la paciente se fucron demeritando en tal forma, que practicado el tratamiento quirúrgico (resección intestinal con enteroanastomosis), la conferma sucumbió.

Debomos lógicamente tconsiderar este caso en forma especial desde el punto de vista estadistico y concluir que la mortalidad en los úlimos cinco años por cmbarazo ectópico, ha sido de cero en el Instituto Materno e Infantil de Bogotá

Vale la pena comparar este dato con los dades por autores extranjeros: Argonz (Op. cit.), Profesor de la Facultad de Medicina de Rosario, Argentina, 
refiriéndose a la mortalidad por embarazo ectópico dice: "La operación antes de las complicaciones debe dar una mortalidad prácticamente cero; las intervenciones durante los accidentes, dan una mortalidad desigual, que depende de la gravedad de la complicación y de otres factores circunstanciales, entre 2 y $5 \%$.

W. O. Johnson (i) del Departamento de Ginecología de la Universidad de Louisville, presentó en abril de 1952 un trabajo ante la Sociedad de Obs. tetricia y Ginecología de la misma ciudad, en el cual reunió 245 casos con una mortalidad de $2.4 \%$.

Priddle, Moulton y Dennis (8), de Detroit, realizaron un estudio de 136 casos a partir de 1949 y dan un porcentaje de mortalidad de $2.9 \%$.

Anatomía Patológica- Hemos considerado de gran importancia hacer en el presente resumen las siguientes observaciones: se han incluído en este trabajo, muchos cásos de embarazo ectópico sin comprobación anatomopatológica. Ello indudablemente, implica una gran falla. Nos hemos atenido únicamente, al diagnóstico y hallazgos quirúrgicos; no se justifica en la actualidad que ello suceda por cuanto se presta a errores. Con mucha frecuencia, al estudiar anatomopatológicamente piczas obtenidas en actos quirúrgicos, se comprueba la diferencia entre el diagnóstico macroscópico del cirujano y el microscópico del Patólogo.

Entre los casos citados en las historias clínicas como de embarazos ectópicos, descartamos aquellos que en nuestro concepto, no tuvieron respaldo del laboratorio y que se prestaban a confusión. Tal cosa hicimos por ejemplo, con varios casos de hematosalpinx. Una simple salpingitis aguda hemorrágica puede confundirse macroscópicamente con un embarazo ectópico. En cuanto a algunos $\mathrm{em}$ barazos ovíricos también los hemos excluído, por la misma causa. El diagnóstico macroscípico de esta variedad de cctópico, no se debe tener en cuenta sino en taras ocasiones; la ruptura de un foliculo de De Graff, los quistes ováricos hemorrágicos, etc., son muchas veces macroscópicamente semejantes.

El interés del estudio anatomopatologico sc pucde demostrar con el caso de la historia clínica 360 del año de 1953: En esta paciente se hizo el diagnóstico clínico de embarazo cetópico no roto; al practicar la intervención quirúrgica se encontró: un embarazo cctópico en evolución implantado en la trompa derecha, un quiste ovárico del mismo lado, la trompa izquierda congestionada y con un quiste entre sus limbrias y sobre la cara anterior del ligamento ancho, y adherido a ella, un huevo abortado. Al efectuar los estudios microscópicos, se comprobó la presencia de embarazo ectópico bilateral, caso por lo demás, de excepción.

\section{CASOS CLINICOS ESPECIALES}

Al lado de la historia clinica citada antes, hemos considerado oportuno, mencionar a continuación la historia de dos pacientes cuyos casos clínicos, por su rara ocurrencia, y las conclusiones que de ellas se pueden obtener, les con- 
fiere particular interés. La primera de ellas fuc publicada por nosotros (") en el número $1^{\text {9 }}$ de la Revista del Hospital de San Juan de Dios.

Historia $N^{\text {yo }} 2.114$ de 1952 :

N.N. de 26 años. Natural de Natagamim. Ingresat al Servicio por que desde hace 15 dias no siente los mowmientos del feto.

Antecedentes familiares: La midre ex demente

Antecedentes persomales a Conecologicos: Menarquia a los 13 años, ciclo menstrual irregular con 3 dias de duracion: $b$ Patologicos: enfermedades de la infancia; c)-Quirúgicos: apendicictomia y colecistectomia; d)-Obstétricos: dos partos espontáneos a termino con fetos vivos. Exámenes serológicos y radioscopia pulmonar: negativos.

\section{Ultima menstruación: 20 de enero 52}

Durante el embarazo ha prescriado: náuscas, vómito, mareos, cefalalgia, vá rices de los miembros inferiores, al iàs diversás e insomnio.

Examen general: Paciente con estado general satisfactorio. Tensión arterial Mx $130 \mathrm{Mn} 90$. Corazón normal bon 68 pulsaciones. Dientes en mal estado. Pulmones, aparato digestivo y urinario: normales.

Examen ohstétrico: Sobre la pared abdominal se observan las cicatrices qui rúrgicas de apendicectomia y colecistectomia, es una pared de tonicidad normal. La altura uterina es de 30 cms.; su consistencia es normal y su superficie no of re ce nada de particular. Al estudiar el contenide uterino, se encuentra un feto en situación transwersa, con su polo cefílico hacia el flanco izquierdo con signo de crepitacion fácilmente apreciable. La auscultacion fetal es negativa.

Tacto raginal: Genitales externes normales. Cuello central, largo, blando, con orificio externo entreabierto. In los fonder de saco, nada especial.

La paciente manifiesta no haber cenide delores y la única causa de su in greso al Il lospital es la aunencia de movimicnton letales desde hace 15 días.

Evolucan: 1121 de octubre se decide provecar el trabajo del parto, en vista de que no se ha presentado espontaneamente. (on este fin se administran $5 \mathrm{ll}$. de Pitocin en 500 c.e. de solución de dextrosia al 5\%, gota a gota. La paciente no manifiesta trastorno alguno y clinicamente, tampoco se aprecian contracciones uterinas.

El fracaso en la inducción se atribuye a falta de preparación de la paciente, razon por la cual, se procede en los dias siguientes a sensibilizar el músculo uterino mediante la administracion repetida de foliculina. Cuatro días más tarde, el 28 de octubre, se dan a la paciente 30 gramos de aceite de ricino, se aplica enema bubonoso y se dan dosis repetidas de quinina para intensificar luégo la induc.

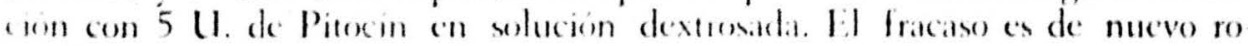
eunde y se resuclec pener a la paciente en reperso durante varios dias.

El $12 \mathrm{de}$ noviembre, se practica un nucvo examen obstétrico; llama la aten ción el grado de retraccion de la matriz que impide la percepción de partes fe 
tales. Al tacto vaginal se encuentra el cuello muy alto, de consistencia fibrosa y francamente tubular.

Insistiendo en la inducción, se practica taponamiento cervical; se administra nuevamente metrazol-quinina y-luégo pitocín en solución de dextrosa. De nucvo observamos con sorpresa, la ineficacia del sistema.

El 15 de noviembre, se solicita estudio radiográlico con el fin de aportar nuevos datos a la historia clínica. El resultado es cl siguiente: "Embarazo con feto único en presentación cefálica y dorso a la derecha. Ilay cabalgamiento de los huesos del cráneo y deformaciones del rapuis que hacen pensar en feto 'muerto". Fdo., C. Sienna E.

En estas condiciones, el 22 de noviembre se decide intervenir quirúrgica. mente a la paciente, practicando una cesárea por vía-viginal.

Intervención quirúrgica.

Anestesia raquídea con Scurocaine.

Se inició la intervención con el descenso del cuello traccionando con pin zas: esta maniobra fue difícil ya que se encontraba en situación perfectamente retropúbica y dirigido hacia adelante. Se hizo sección transversal y despegamiento de la mucosa vaginal anterior. En este momento, observando los caracteres del cuello, se encontró perfectamente cerrado siendo necesaria la dilatación intrumental mediante bujía de Hegar. Introducido un dedo a través del cuello se encontró la cavidad uterina vacía; se pasó el histerómetro que penetró hasta el número 14. En estas circunstancias, se sospecthó algo no previsto, decidiéndose la intervención por vía alta.

Al hacer la laparotomía supra e infraumbilical, se encontró la pared rica mente vascularizada; el peritóneo apareció fuertemente engrosado. En la cavi dad abdominal, pequeño derrame de aspecto serose, y ocupándola, una masa mediana que se pensó fuese el utero con adlerencias fácilmente liberables a la serosa parietal y en algunas zonas pecuecinas, al mesenterio. Dicha masa pre sentaba caracteres fibrosos; haciendo sobre su pared una incision mediana se obervó que no sangraba; de su interior fluyó liquido de color amarillo verdoso c introducida la mano, se extrajo un feto muerto y macerado, correspondiendo por su tamaño a una gestación a término.

Al tratar de extraer la placenta, y revisando cuidadosamente el organo, se encontró que en realidad se trataba del saco ovular colocado precisamente sobre el fondo del útero y comunicando con su cavidad a nivel de la porción inters ticial de la trompa izquierda por un orificio de medio a 1 centimetro de diame tro. Dicho saco presentaba un espesor aproximado de medio centímetro, aun que en algunas zonas era mas delyado. La individualización del anexo izquier do fue imposible por hallarse englobado en la pared ovular; no sucedió asi con el del lado derecho que se encontro perfectamente libre y en situación normal. La placenta, delgada pero extendida, se implantaba exactamente sobre el fon do uterino y contraía con éste, intimas relaciones. El útero en sí, se presentaba desviado un poco hacia la derecha y aumentado moderadamente de tamaño; a 
aivel de su comunicación con el quiste fetal, el miometrio emergia para hacer parte de la pared ovular.

Se procedió entonces a extrace d saco ovular y como se continuara sin linea de separación con el fondo uterino, se decidio practicar histerrectomía fundica conservando el anexo derecho. Hecha la rescicion. se procedió a la sumura del útero en trés planos. Practicada la peritonizacions se realizó el cierre d. lis pared.

Durante la intervencion fue necesaria la anestesia adicional con ciclo pano éteroxigeno y para contrarrestar la hemorragia se administraron 500 c.c. singre total.

Practicada la operación la paciente se encontraba en estado de shock y fue nccesaria la administración de cardiotónieos, hipertensores, etc.

Hasta aquí la primera historia clínica. El hallazgo de un embarazo extrauterino a término es en realidad de verdad una eventualidad muy rara. Si a ello agregamos el que la modalidad de ese embarazo sea la de una gestación TUBO1NTERSTICIAL, el hecho es mucho más interesante puesto que su frecuencia afrancamente excepcional. Los casos registrados en la literatura obstétrica son "uraordinarios.

A pesar de ser el embarazo abdominal una variedad de las más raras dentro us extrauterinos, es el intersticial por su misma patogenia, el que ocupa uno d. los primeros lugares por su escasa frecuencia.

Nuestro diagnóstico se hizo, como sucede en casos similares, tardiamente. Y a que ocurre que cuando un embarazo extrauterino llega hasta el final, hay una sric de factores que hacen muchas veces imposible su reconocimiento; esto es mucho más apreciable cuando no sólo el cmbarazo ha llegado a su término, sino además el feto ha muerto varios dias antes de ser examinadas la paciente pur primera vez. Tal cosa ocurrici con nuestra enferma. A la palpación de su ab. thum, no se encontraron las irregularidades de la masa que lo ocupaba; tam- existieron los fenomenos que constituyen el falso trabajo, pues la paciente só al servicio únicamente por haber dejado de percibir los movimientos del frcte. La serie de trastornos digestivos que sobrevienen como consecuencia de estos embarazos, no se presentaron. La misma situación del sico ovular precisamente sobre el fondo del útero, no permitia siquiera dudar de que se tratara de 1. matriz en gestación. El estudio radiográfico, que scgún Brindeau muestra al feto en situación más alta de lo normal y retraido sobre si mismo, no dio en nuestra paciente otros signos que los encontrados en un embarazo común y coriente con feto muerto.

Un signo propio de estos combarazos y que se aprecia al tacto vaginal es el aso reblandecimiento del cuelle; hallado en el eximen de nuestra enferma - dio una interpretación diferente; se penso que se trataba de un fibrosis avical, hipótesis que se reforzi con la ausencia de respucsta al estímulo de los xitócicos por parte del útero. Esto fue precisamente lo que hizo que en la terera inducción al trabajo, se practicara taponamiento cervical. 
Historia No 4.188 de 1953 :

N. N. de 24 años. Multigestante V.

Sin antecedentes hereditarios ni personales importantes.

Los cuatro embarazos anteriores normales, con partos a tumino-espontá. neos y con fetos vivos.

Ultima menstruación: febrero de 1953.

Desde mediados de abril presentó hemorragias genitales repetidas con ex pulsión de coágulos $\mathrm{e}$ indoloras.

El 22 de junio se hospitalizó en el Instituto y fuc tratada como si fuese una amenaza de aborto con embarazo de 4 a $41 / 2$ meses. Diez, siete dias más tarde habiendo cesado la hemorragia salió del Servicio.

El 25 de noviembre, reingresó nuevamente con hemorragia. At practicar el examen se encontró: útero hipertónico e irregular con una alfura de 24 cms. Al tacto vaginal: el cuello central, largo, reblandecido. La irrugularidad de ta superficie del útero se atribuyó a fibromatosis. La auscultacion fetal fue nega tiva. Se pidió estudio radiográfico del contenido uterino; el re ultado es el siguiente: "Existe un feto deformado al parecer muerto". (Fdo.). E. Siema G,

En estas condiciones, se practicaron en los dias siguientes, tres induccio nes al trabajo con fracaso rotundo en todas ellas y habiendo hecho taponamiento cervical en la tercera. Días más tarde, la paciente se quejó de cólicos abdo. minales y presentó vómito vilioso. La palpación del abdomen despertaba gran dolor; apareció hipertermia.

Bajo anestesia general se procedio a dilatar el cuello artificialmente. El di latador no alcanzó el orificio interno; sobrevino copiosa hemorragia que obligó a dejar un taponamiento cervical micntras se hacia laparotomia.

En esas condiciones tuvimos la oportunidad de examinar detenidamente a la paciente. Nos llamó la atención en la anamnésis la sintomatología netamente hemorrágica que acompanó al embarazo desde sus comienzos, así como también los caracteres de ella: repetida e indolora. En segundo lugar, la palpa. ción abdominal cra muy dolorosa y acompañada de cierto grado de defensa muscular. La masa intrabdominal no era ovoide como correspondia a una ges tación de esa edad ( 7 meses aproximadamente por el tamaño), sino globulosa. La palpación del feto era impracticable puesto que la retracción de la masa sobre él, lo impedia; pero hacia la fosa iliaca derecha algo crepitaba.

El tacto vaginal nos reveló la existencia de un cucllo en situación sensible. mente normal, pero cuya longitud no estaba en relación con la edad del emba. razo, puesto que alcanzaba unos 8 ctms. El dato de haber sido imposible dilatar el orificio interno, hacia más extraño el cuadro clínico. Los resultados del exa. men anterior y el fracaso en tres inducciones, nos hicieron pensar en la posibilidad de un embarazo ectópico avanzado.

Horas más tarde, la paciente fue intervenida. El resultado de la interven ción, de acuerdo con la descripción de los cirujanos es la siguiente: "Se encontró 
un embarazo extrauterino con un gran saco adherido a la matriz que se hallaba lineramente aumentada de tamano y adherida a la cara anterior del saco ectópico. H.bia además adherencias al epiplón y a las asas intestinales. Poco a poco se fueron liberando las múltiples adherencias del saco hasta dejarlo prácticamente independiente. En el curso de las maniobras se abrió dicho saco saliendo liquido purulento. En vista de lo anterior se aspiré su contenido líquido y luégo se exnajo un feto muerto, femenino y a continuación la placenta que salio sin difi cultad. Se procedio entonces a resecar el saco lo cual se logro casi en su totali d.ul quedimdo tan solo una pequena porción adherida al asa sigmoide la cual “ dreno con dren de caucho que se abocó a la herida cutánea. Al revisar la matriz se encontró una perforación en la cara posterior, aproximadamente de $1 \mathrm{ctm}$. ke diametro, la cual se suturó y peritonizó quedando en buenas condiciones; se puso otro dren a partir del fonde del saco de Douglas que también se abocó a la herida cutánea. El saco ectopico se había formado a expensas de la trompa derecha y se extirpó también el ovario del mismo lado quedando el otro anexo en su lugar". En la actualidad esta paciente permanece hospitalizada en el Instituto.

A la historia clínica anterior, poco tenemos que comentarle. La literatura obstétrica relata con alguna frecuencia casos similares. La confusión diagnóstica no es cosa excepcional y una de sus causas es precisamente la escasa frecuencia de estos casos.

Paúl O' Connell $\left({ }^{10}\right)$ del Hospital Sloane y de la Universidad de Columbia describió en junio de 1952 un caso clínico en el cual predominó la sintomatolologia urinaria. Un accidente de obstrucción intestinal determinado al practicar una versión por maniobras externas (el feto se hallaba en siuación transversa), impuso la intervención quirúrgica durante la cual se encontró un cmbarazo tubarico a término.

Juan Gómez Peña y otros (") del Hospital Civil de Maracay relató un caso (n) cl cual se practicaron tres inducciones e inyección intraovular de suero hiper único para provocar el parto de un feto muerto. En vista del fracaso, hicieron laparotomía y hallaron un embarazo tubárico gemelar con fetos de 3.340 y 120 gramos.

\section{SUMARIO}

$1^{\circ}$ - Se hace un resumen de los casos de embarazo ectópico atendidos en cinco años en el Instituto Matemo-Infantil "Concepción Villaveces de Acosta" de Bogotá.

$2^{\circ}$ - Se exponen los porcentajes de frecuencia, edad, paridad, tiempo de amenorrea, sintomatologia, diagnóstico, localizacion, pronóstico y mortalidad.

3?-Se hacen algunas observaciones acerca de la colpotomía posterior, ana tomía patológica y medios biologicos de diagnóstico.

40-Se comentan las intervenciones quirúrgicas, los hallazgos y concomi tancias.

50- - Se describen dos casos de embarazo ectópico avanzado y se hacen al gunos comentarios a ellos. 


\section{CONCLUSIONES}

10-El embarazo ectópico es una entidad cuyo porcentaje de frecuenci se ha conservado semejante en los últimos años a excepción del año de 195 en que su disminución fue notoria.

2?-La mayor frecuencia se ha presentado entre los 24 y los 34 años en pacientes con dos o más gestaciones. favorable.

3?-El porcentaje de mortalidad por embarazo ectópico es francament

4? - El número de casos diagnosticados permite concluír que su estudi cínico ha sido acertado.

50--Los estudios complementarios como las pruebas biológicas y otro exámenes de laboratorio realizados con mayor intensidad pueden mejorar indice diagnóstico.

6?-La colpotomía posterior es una ayuda clínica muy valiosa y su emple ha de hacerse más frecuente.

70- Los estudios anatomopatológicos deben aumentar. Ellos aclaran en oc siones el diagnóstico y permiten sacar conclusiones estadísticas más exact

$8^{\circ}$ - Las pacientes en estado de shock por ruptura de su gestación ectópic corren menos riesgo intervenidas precozmente.

$9^{\circ}$ - La colaboración del Banco de Sangre ha sido decisiva en los casos i icrvenidos y en mucho se debe a él la bondad del porcentaje de mortalid materna.

$10^{\circ}-$ No es conveniente practicar simultáneamente intervenciones quir gicas tales como apendicectomía, durante la operación por ectópico; porq s. une un riesgo quirúrgico más por la posibilidad de contaminación peritone.

$11^{\circ}$ - La anestesia ráquídea sólo puede emplearse en casos especiales.

12?-El fracaso en inducciones repetidas, debe hacer que las pacientes (studicn muy cuidadosamente.

13?-Los síntomas y signos descritos por diversos autores en los casos cmbarazo ectópico avanzado, tienen un valor relatiro; no constituyen úni bases de diagnóstico.

14?-El diagnóstico positivo de ectópico avanzado es a veces, un hallaz quirúrgico, en especial cuando la muerte del feto ha ocurrido tiempo atrás.

150-Las historias clínicas deben mejorarse. Su interés no ha sido suficie temente valorado y ellas constituyen una gran base científica. 


\section{B I B I O G A F I A}

1. -Archila Aguilera Enrique: "El embarazo Extrauterino. - Comentarios a 8 años de Estadística del Hospital de San Juan de Dios de Bogotá". Tesis de grado. 1946. Página 18.

2.-Góngora Sánchez Humberte: "Estudio Clínico del Embarazo Ectópico". Tesis de grado. 1952. Página 10.

3.-Novoa Rodriguez. Luis: "Estudio Clínico-Estadístico del Embarazo Extrauterino". Tesis de grado. 1953. Página 12.

4.-De Lee Greenhill: "Principios y Práctica de Obstetricia”. Vol. I, páginas 499, 502. 8a Ed. Editorial Hispano Americana, México, 1945.

5.-Nubiola-Zárate: "Tratado de Obstetricia". Vol. II, página 569. 19 Ed. Editorial Labor. Barcelona, 1951.

6.-Argonz y Ricci: "Tratado de Obstetricia” de Nubiola. Páginas 569, 565.

7.-Johnson W. O.: "A Study of Tow hundred forty five cases of Ruptured Ectopic Pregnancy". Am. J. of Obst. and Gyn. 64: 1.102, Nov. 1952.

8.-Priddle, Moulton y Denis: "Ectopic Pregnancy". Am. J. Obst. and Gyn. 64: 1.093, Nov. 1952.

-Gómez Palacino J. A.: "Un Embarazo Tubo-Intersticial a Término". Revista del Hospital de San Juan de Dios, Bogotá, 1: 101. Enero, 1953.

10.-O'Con Nel C. Paúl: "Full-term Tubal Pregnancy". Am. J. Obst. and Gyn. 63: 1.305, June, 1952.

11. -Gómez Peña Juan: "Un Caso de Embarazo Tubario Gemelar a Término". Revista de la Sociedad de Obstetricia y Ginecología de Venezuela. Tomo XIII: $376,1953$. 